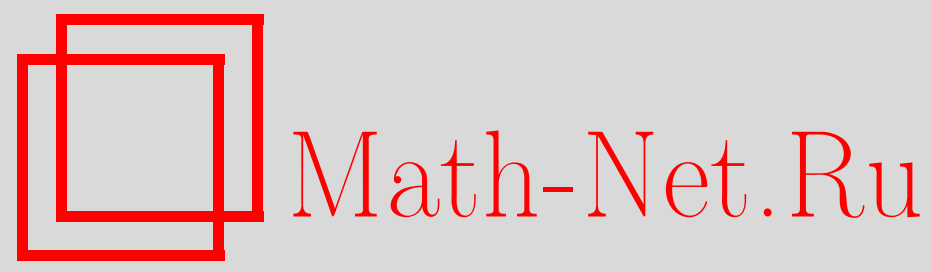

А. Ю. Колесов, Диффузионная неустойчивость однородного цикла, бифурцирующего из петли сепаратрисы, Матем. заметки, 1998, том 63, выпуск 5, 697-708

DOI: https://doi.org/10.4213/mzm1336

Использование Общероссийского математического портала Math-Net.Ru подразумевает, что вы прочитали и согласны с пользовательским соглашением http://www.mathnet.ru/rus/agreement

Параметры загрузки:

IP : 54.89 .56 .158

26 апреля 2023 г., 18:29:30

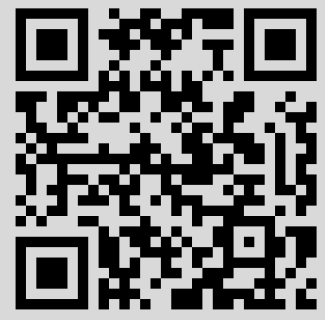


УДК 517.926

\section{ДИФФУЗИОННАЯ НЕУСТОЙЧИВОСТЬ ОДНОРОДНОГО ЦИКЛА, БИФУРЦИРУЮЩЕГО ИЗ ПЕТЛИ СЕПАРАТРИСЫ}

\section{А. Ю. Колесов}

Рассматривается краевая задача

$$
\frac{\partial u}{\partial t}=D \frac{\partial^{2} u}{\partial x^{2}}+F(u, \mu),\left.\quad \frac{\partial u}{\partial x}\right|_{x=0}=\left.\frac{\partial u}{\partial x}\right|_{x=\pi}=0 .
$$

Здесь $u \in \mathbb{R}^{2}, D=\operatorname{diag}\left\{d_{1}, d_{2}\right\}, d_{1}, d_{2}>0$, а гладкая по совокупности переменных вектор-функция $F$ такова, что при $0<\mu \ll 1$ у краевой задачи имеется однородный (не зависящий от $x$ ) цик л, бифурцирующий из петли сепаратрисы седла. Устанавливаются условия устойчивости и неустойчивости этого цикла и дается их геометрическая интерпретация.

Библиография: 8 названий.

Параболические системы типа реакция-диффузия с граничньми условиями Неймана, имеющие однородный (не зависящий от пространственной переменной) цикл, встречаются во многих приложениях [1], [2]. Вопрос же об устойчивости однородного цикла актуален в связи с тем, что наряду с известной теоремой Тьюринга-Пригожина [3] он объясняет природу возникновения пространственно неоднородных структур в однородных средах. К настоящему времени этот вопрос изучен для случаев, когда однородньй цикл близок к гармоническому или является релаксационным (соответствующие результаты были получены в [4] и впоследствии вошли в монографии [5], [6]). Ниже данньй вопрос исследуется для нового класса систем, однородньй цикл которых бифурцирует из петли сепаратрисы седла.

1. Постановка задачи. На отрезке $0 \leqslant x \leqslant \pi$ рассмотрим краевую задачу

$$
\frac{\partial u}{\partial t}=D \frac{\partial^{2} u}{\partial x^{2}}+F(u, \mu),\left.\quad \frac{\partial u}{\partial x}\right|_{x=0}=\left.\frac{\partial u}{\partial x}\right|_{x=\pi}=0,
$$

где $u \in \mathbb{R}^{2}, D=\operatorname{diag}\left\{d_{1}, d_{2}\right\}, d_{1}, d_{2}>0$, а вектор-функция $F$ принадлежит классу $C^{\infty}\left(\mathbb{R}^{2} \times\left[0, \mu_{0}\right] ; \mathbb{R}^{2}\right), \mu_{0}>0$. В качестве фазового пространства (пространства начальньх условий $u(0, x))$ краевой задачи (1) возьмем гильбертово пространство $E=$ $W_{2}^{1}\left([0, \pi] ; \mathbb{R}^{2}\right)$.

Дальнейшие ограничения, накладываемые на задачу (1), связаны с системой на плоскости

$$
\dot{u}=F(u, \mu) \text {. }
$$

Работа выполнена при финансовой поддержке Российского фонда фундаментальных исследований, грант № 96-01-00207. 
УСловиЕ 1. Считаем, что при $\mu=0$, во-первых, система (2) имеет седловое нулевое состояние равновесия, причем собственные значения - $\lambda_{-}, \lambda_{+}$матрииы $A_{0}=\partial_{u} F(0,0)$ таковы, что $0<\lambda_{+}<\lambda_{-}$; во-вторых, имеется петля сепаратриcbl $\Gamma_{0}$ (cм. puc. 1, 2).

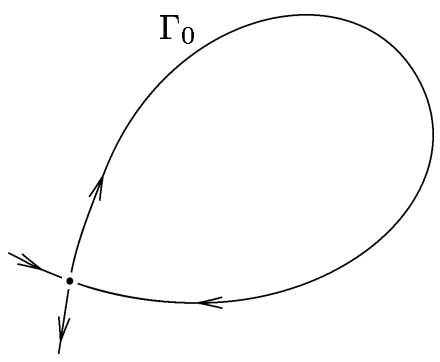

Рис. 1

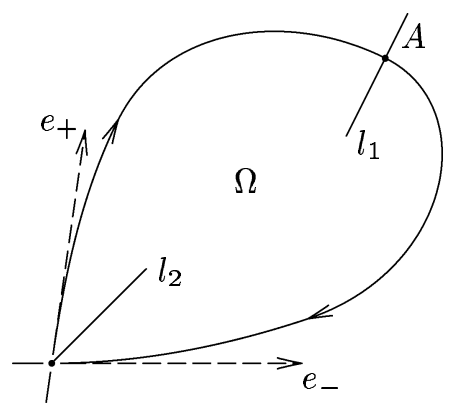

Рис. 3

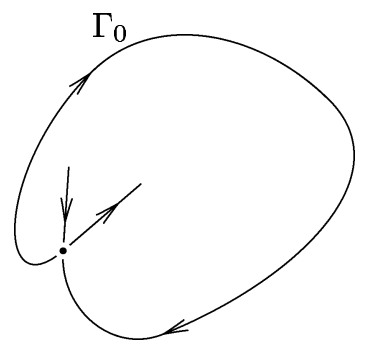

Рис. 2

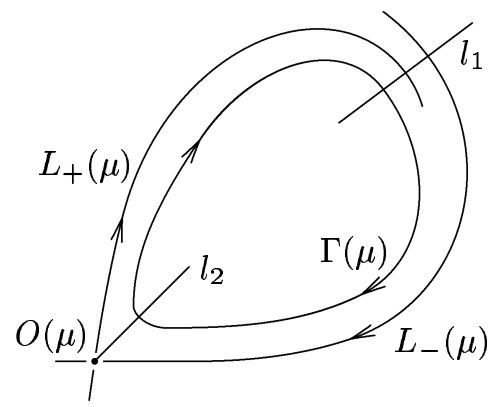

Рис. 4

Для формулировки следующего условия фиксируем произвольную точку $A \in \Gamma_{0}$, $A \neq 0$, и рассмотрим отрезок нормали $l_{1}$ к кривой $\Gamma_{0}$, проходяший через эту точку (см. рис. 3). В качестве параметра $s$ на $l_{1}$ возьмем евклидово расстояние до точки $A$. При этом в случае петли $\Gamma_{0}$, изображенной на рис. 1 , указанное расстояние берется со знаком “+” по направлению внутренней нормали к области $\Omega$ (см. рис. 3 ), ограничиваемой кривой $\Gamma_{0}$, и со знаком “-” в противоположном направлении. В случае же петли $\Gamma_{0}$, показанной на рис. 2, знаки параметра $s$ изменяются на противоположные.

При $0<\mu \ll 1$ нулевое состояние равновесия системы $(2)$ переходит в седловое состояние равновесия $O(\mu)$, устойчивая $L_{+}(\mu)$ и неустойчивая $L_{-}(\mu)$ сепаратрисы которого, вообще говоря, уже не образуют петлю (см. рис. 4). Обозначим через $s_{+}(\mu)$ и $s_{-}(\mu)$, $s_{ \pm}(0)=0$, соответственно координаты точек их пересечения с трансверсалью $l_{1}$ и положим $\varkappa(\mu)=s_{+}(\mu)-s_{-}(\mu)$.

УСловие 2. Предполагаем, что

$$
\varkappa_{0}^{\prime}=\left.\frac{d \varkappa}{d \mu}\right|_{\mu=0}>0 .
$$

Как известно [7], условия 1, 2 гарантируют существование у системы (2) при всех $0<\mu \ll 1$ единственного экспоненциально орбитально устойчивого цикла (см. рис. 4)

$$
\Gamma(\mu)=\left\{u: u=u_{0}(t, \mu), u_{0}(0, \mu) \in l_{1}\right\}
$$


бифурцируюшего из петли $\Gamma_{0}$. В дальнейшем, однако, нам потребуются более детальные его асимптотические свойства. Для их выявления рассмотрим собственные векторы $e_{+}$и $e_{-}$матрищы $A_{0}$, отвечающие ее собственньм значениям $\lambda_{+}$и $-\lambda_{-}$, причем считаем, что направление $e_{+}$совпадает с движением фазовой точки по кривой $\Gamma_{0}$, а направление $e_{-}$ему противоположно (см. рис. 3 ). Введем в рассмотрение выходящий из начала координат по направлению $e_{+}+e_{-}$достаточно мальй отрезок $l_{2}$ (см. рис. 3 ) и обозначим соответственно через $-\tau_{1}(\mu)$ и $\tau_{2}(\mu)$ первые отрицательный и положительный моменты времени такие, что

$$
u_{0}\left(-\tau_{1}(\mu), \mu\right) \in l_{2}, \quad u_{0}\left(\tau_{2}(\mu), \mu\right) \in l_{2}
$$

Тогда, очевидно, период $T(\mu)$ цикла (3) равен $\tau_{1}(\mu)+\tau_{2}(\mu)$.

ЛЕмма 1. Справедливы асимптотические формуль

$$
\tau_{1}(\mu)=\frac{\sigma_{0}}{\lambda_{+}+\lambda_{-}} \ln \frac{1}{\mu}+O(1), \quad \tau_{2}(\mu)=\frac{1}{\lambda_{+}+\lambda_{-}} \ln \frac{1}{\mu}+O(1)
$$

и равномерное по $-\tau_{1}(\mu) \leqslant t \leqslant \tau_{2}(\mu)$ асимптотическое представление

$$
u_{0}(t, \mu)=u_{0}(t)+O\left(\mu^{\gamma}\right)
$$

где $\sigma_{0}=\lambda_{-} / \lambda_{+}, \gamma=\sigma_{0} /\left(\sigma_{0}+1\right), u_{0}(t)$ - решение системы (2) при $\mu=0$, отвечающе петле $\Gamma_{0}$.

ДокАЗАТЕЛЬСтво. Проясним сначала сам факт существования цикла (3). С этой целью будем считать, что на секущей $l_{1}$ при $\mu>0$ выбран тот же самьй параметр $s$, но теперь значению $s=0$ соответствует точка $l_{1} \cap L_{-}(\mu)$. Тогда в силу условия 1 на этой секущей при $0 \leqslant s \leqslant s_{0}$, где $s_{0}>0$ достаточно мало, определено отображение последования П по траекториям системы (2). Покажем, что для него имеет место представление

$$
\Pi: s \rightarrow \varkappa(\mu)+c(\mu) s^{\sigma(\mu)}+\varphi(s, \mu),
$$

где $\varkappa(\mu)$ - функция из условия $2 ; c(\mu), \sigma(\mu)$ - некоторые функции класса $C^{1}$ такие, что $c(0)>0, \sigma(0)=\sigma_{0}$; для непрерьвно дифференцируемого по $s, \mu$, остатка $\varphi$ при $s \rightarrow+0$ равномерно по $\mu$ справедливы равенства

$$
\varphi(s, \mu)=o\left(s^{\sigma_{0}}\right), \quad \partial_{s} \varphi(s, \mu)=o\left(s^{\sigma_{0}-1}\right) .
$$

Обоснование всех перечисленных свойств базируется на известном [8] факте о существовании замены переменных класса $C^{1}$, приводящей в некоторой окрестности седла систему (2) к линейной нормальной форме. Для более детального описания этой замены, считая (это не ограничивает общности), что $F(0, \mu) \equiv 0$, введем в рассмотрениегладкие по $\mu$ собственные векторы $e_{+}(\mu), e_{-}(\mu)$ матрицы $\partial_{u} F(0, \mu)$, отвечающие ее собственным числам $\lambda_{+}(\mu),-\lambda_{-}(\mu)$ и обрашающиеся при $\mu=0$ во введенные вьше собственные векторы $e_{+}$и $e_{-}$матрицы $A_{0}$. Тогда интересующая нас замена может быть представлена в виде

$$
u=G(\mu) y+H(y, \mu), \quad y=\operatorname{colon}\left(y_{1}, y_{2}\right),
$$

где $G(\mu)=\left[e_{-}(\mu), e_{+}(\mu)\right]$, а непрерьвно дифференцируемая по совокупности переменных вектор-функция $H$ такова, что $H(0, \mu) \equiv 0, \partial_{u} H(0, \mu) \equiv 0$. Результатом же ее применения к системе (2) служит линейная система

$$
\dot{y}_{1}=-\lambda_{-}(\mu) y_{1}, \quad \dot{y}_{2}=\lambda_{+}(\mu) y_{2} .
$$


Для того чтобы воспользоваться заменой (8) при анализе отображения П, рассмотрим вспомогательные трансверсали $\Sigma_{j}\left\{\left(y_{1}, y_{2}\right): y_{j}=q\right\}, j=1,2$, где $q>0$ достаточно мало. В качестве параметра $s$ на $\Sigma_{1}$ и $\Sigma_{2}$ возьмем соответственно $s=y_{1}$ и $s=y_{2}$. Представим, далее, П в виде $\Pi_{3} \Pi_{2} \Pi_{1}$, где отображение $\Pi_{1}$ действует из $l_{1}$ в $\Sigma_{1}, \Pi_{2}-$ из $\Sigma_{1}$ в $\Sigma_{2}, \Pi_{3}-$ из $\Sigma_{2}$ в $l_{1}$, и проанализируем каждое из этих отображений в отдельности.

Решая систему $(9)$, сингулярную часть отображения П, т.е. $\Pi_{2}$, находим в явном виде:

$$
\Pi_{2}: s \rightarrow q^{1-\sigma(\mu)} s^{\sigma(\mu)}, \quad \sigma(\mu)=\frac{\lambda_{-}(\mu)}{\lambda_{+}(\mu)} .
$$

Отображения же

$$
\Pi_{1}: s \rightarrow f(s, \mu), \quad \Pi_{3}: s \rightarrow g(s, \mu)
$$

регулярны, так как время, в течение которого по траекториям системы (2) попадаем из $l_{1}$ на $\Sigma_{1}$ или из $\Sigma_{2}$ на $l_{1}$, имеет порядок 1 (при $s, \mu \rightarrow 0$ оно стремится к соответствующему времени для траектории $\Gamma_{0}$ ). Отсюда и из обших свойств решений систем на плоскости заключаем, что функции $f, g$ непрерьвно дифференцируемы по совокупности переменных и, кроме того,

$$
f(0, \mu) \equiv 0, \quad \partial_{s} f(0,0)>0, \quad g(0, \mu)=\varkappa(\mu), \quad \partial_{s} g(0,0)>0 .
$$

Остается добавить, что свойства (6), (7) отображения П вытекают из (10)-(12) очевидным образом.

Учитывая в $(6),(7)$ неравенства $\sigma_{0}>1, \varkappa(\mu)>0$ (см. условия 1,2$)$, убеждаемся, что на отрезке $0 \leqslant s \leqslant s_{0}$ отображение П имеет единственную экспоненциально устойчивую неподвижную точку

$$
s=s_{*}(\mu): s_{*}(\mu)=\varkappa_{0}^{\prime} \mu+O\left(\mu^{r}\right), \quad r=\min \left\{2, \sigma_{0}\right\},
$$

соответствующую циклу $\Gamma(\mu)$.

Для обоснования асимптотических равенств (4) рассмотрим участок цикла $\Gamma(\mu)$, лежаший между $\Sigma_{1}$ и $\Sigma_{2}$ и задаваемьй в переменных $\left(y_{1}, y_{2}\right)$ формулами

$$
y_{1}=q \exp \left(-\lambda_{-}(\mu) t\right), \quad y_{2}=y_{*}(\mu) \exp \left(\lambda_{+}(\mu) t\right)
$$

где $0 \leqslant t \leqslant \bar{\tau}_{1}(\mu)$

$$
y_{*}(\mu)=f\left(s_{*}(\mu), \mu\right) \sim \partial_{s} f(0,0) \varkappa_{0}^{\prime} \mu, \quad \mu \rightarrow 0, \quad \bar{\tau}_{1}(\mu)=\frac{1}{\lambda_{+}(\mu)} \ln \frac{q}{y_{*}(\mu)} .
$$

Заметим, далее, что хотя в переменных $\left(y_{1}, y_{2}\right)$ отрезок $l_{2}$ искривляется, при нахождении главной асимптотики $\tau_{1}(\mu), \tau_{2}(\mu)$ его можно заменить на прямую $y_{1}=y_{2}$. Учитьвая это обстоятельство, из (14) находим время

$$
\bar{\tau}_{2}(\mu)=\frac{1}{\lambda_{-}(\mu)+\lambda_{+}(\mu)} \ln \frac{q}{y_{*}(\mu)}
$$

в течение которого, двигаясь по циклу $\Gamma(\mu)$, попадаем из $\Sigma_{1}$ на указанную прямую. И наконец, объединяя (15), (16) с очевидными равенствами

$$
\tau_{1}(\mu)=\bar{\tau}_{1}-\bar{\tau}_{2}+O(1), \quad \tau_{2}(\mu)=\bar{\tau}_{2}+O(1),
$$

убеждаемся в справедливости формул (4). 
Из равенства (13) для начального условия цикла (3) имеем

$$
u_{0}(0, \mu)=u_{0}(0)+O(\mu) .
$$

А отсюда автоматически следует, что при любых фиксированных $t_{1}, t_{2}>0$ равномерно по $t \in\left[-t_{1}, t_{2}\right]$

$$
u_{0}(t, \mu)=u_{0}(t)+O(\mu) .
$$

Остаток же в формуле (17) “портится" лишш в окрестностях точек $t=-\tau_{1}(\mu)$ и $t=\tau_{2}(\mu)$, когда функции (14) приобретают порядок $\mu^{\gamma}$. Лемма 1 доказана.

Итак, при вьполнении условий 1,2 и при $0<\mu \ll 1$ краевая задача (1) имеет пространственно однородный цикл (3), устойчивый по отношению к однородным возмущениям (т.е. в рамках системы (2)). Тем самьм, возникает проблема исследования его устойчивости по отношению к произвольным возмушениям, которая и решается в данной работе.

2. Теорема об устойчивости. Условия устойчивости однородного цикла (3) допускают наглядную геометрическую интерпретацию: если при $\mu=0$ неустойчивое многообразие нулевого седлового состояния равновесия системы (1) по-прежнему одномерно, т.е. при учете диффузионных слагаемых у него не появляются дополнительные (пространственно неоднородные) неустойчивые направления, то при $\mu>0$ устойчивым будет и цикл (3). Точнее говоря, справедливо следующее утверждение.

ТЕОрема 1. Пусть выполнены условия 1,2 и все собственные значения матрии,

$$
A_{0}-k^{2} D, \quad k=1,2, \ldots,
$$

имеют отрицательные действительные части. Тогда однородныи иикл (3) краевой задачи (1) орбитально әкспоненииально устойчив (в метрике фазового пространства $E$ ).

ДокАЗАТЕЛЬСТво. Как известно [4]-[6], за устойчивость цикла (3) по отношению к пространственно неоднородньг возмущениям отвечают мультипликаторы систем

$$
\dot{h}=\left(A(t, \mu)-k^{2} D\right) h, \quad A(t, \mu)=\left.\partial_{u} F\right|_{u=u_{0}(t, \mu)}, \quad k=1,2, \ldots,
$$

получающихся из (1) при линеаризации на этом цикле и последующем применении метода Фурье по функциям $\cos k x, k=0,1, \ldots$.

При $\mu=0$ системы (19) переходят в системы

$$
\dot{h}=\left(A(t)-k^{2} D\right) h, \quad k=1,2, \ldots,
$$

в которых при $t \rightarrow-\infty$ и при $t \rightarrow+\infty$ соответственно

$$
A(t)-A_{0}=O\left(\exp \left(\lambda_{+} t\right)\right), \quad A(t)-A_{0}=O\left(\exp \left(-\lambda_{-} t\right)\right) .
$$

Поэтому (см. [8]) главная асимптотика при $t \rightarrow \pm \infty$ решений этих систем определяется из уравнений

$$
\dot{h}=\left(A_{0}-k^{2} D\right) h, \quad k=1,2, \ldots
$$

Отсюда и из гурвицевости матриц (18) для операторов Коши $U_{k}(t, s), U(s, s)=I$ систем (20) следует оценка

$$
\left\|U_{k}(t, s)\right\| \leqslant M \exp (-\delta(t-s)), \quad t \geqslant s .
$$


Здесь $\|\cdot\|$ - фиксированная матричная норма, $\delta \in\left(0, \delta_{0}\right)$, где

$$
\delta_{0}=\min _{k \geqslant 1, j=1,2}\left\{-\operatorname{Re} \lambda_{k}^{j}\right\}>0
$$

a $\lambda_{k}^{1}, \lambda_{k}^{2}$ - собственные числа матриц (18). Символами $M, M_{1}$ и т. д. здесь и ниже обозначаем различные, не зависящие от $t, s, \mu, k$, положительные постоянные, точные значения которых несущественны.

При $\mu>0$, используя вытекающее из (5) асимптотическое представление

$$
A(t, \mu)=A(t)+\mu^{\gamma} \Delta(t, \mu), \quad \max _{-\tau_{1}(\mu) \leqslant t \leqslant \tau_{2}(\mu)}\|\Delta(t, \mu)\| \leqslant M,
$$

для матриц Коши $U_{k}(t, s, \mu)$ систем (19) приходим к интегральным уравнениям

$$
U_{k}(t, s, \mu)=U_{k}(t, s)+\mu^{\gamma} \int_{s}^{t} U_{k}(t, \tau) \Delta(\tau, \mu) U_{k}(\tau, s, \mu) d \tau
$$

Учитьвая, далее, в (24) оценки (22), (23), для

$$
v_{k}(t, s, \mu)=\left\|U_{k}(t, s, \mu)\right\| \exp \delta(t-s)
$$

получаем неравенство

$$
v_{k}(t, s, \mu) \leqslant M_{1}+M_{2} \mu^{\gamma} \int_{s}^{t} v_{k}(\tau, s, \mu) d \tau
$$

из которого, в свою очередь, с помощью леммы Гронуолла вьводим оценку

$$
v_{k}(t, s, \mu) \leqslant M_{3}, \quad-\tau_{1}(\mu) \leqslant s \leqslant t \leqslant \tau_{2}(\mu)
$$

Для завершения доказательства рассмотрим матрицы монодромии

$$
V_{k}(\mu)=U_{k}\left(\tau_{2}(\mu),-\tau_{1}(\mu), \mu\right)
$$

систем (19). Из неравенств (25) и асимптотических формул (4) следует, что

$$
\left\|V_{k}(\mu)\right\| \leqslant M \mu^{\delta / \lambda_{+}}
$$

а значит, при $\mu \rightarrow 0$ равномерно по $k \geqslant 1$ модули всех мультипликаторов этих систем стремятся к 0 . Теорема 1 доказана.

3. Две теоремы о неустойчивости. Перед формулировкой следующей теоремы проделаем ряд вспомогательных построений. Рассмотрим систему

$$
\dot{h}=(A(t)+B(\alpha)) h,
$$

где $B(\alpha)=\operatorname{diag}\{\alpha,-\alpha\}$, а параметр $\alpha$ принадлежит множеству

$$
\Lambda=\left\{\alpha \in \mathbb{R}: \operatorname{det}\left(A_{0}+B(\alpha)\right)<0\right\} .
$$

Обратим внимание, что множество $(27)$ либо совпадает с $\mathbb{R}$, либо получается из $\mathbb{R}$ при выбрасывании некоторого отрезка конечной длины. Рассмотрим, далее, собственные значения $-\lambda_{-}(\alpha), \lambda_{+}(\alpha), 0<\lambda_{+}(\alpha)<\lambda_{-}(\alpha)$ матрицы $A_{0}+B(\alpha)$ и отвечающие им собственные векторы $e_{-}(\alpha), e_{+}(\alpha)$, которые выбираем аналитически зависящими от $\alpha \in \Lambda$ и имеющими единичную евклидову норму. 
Лемма 2. Система (26) имеет аналитически зависящие от $\alpha \in \Lambda$ решения $h=h_{ \pm}(t, \alpha)$ с асимптотикой

$$
h_{ \pm}(t, \alpha)=\left(e_{ \pm}(\alpha)+o(1)\right) \exp \left( \pm \lambda_{ \pm}(\alpha) t\right), \quad t \rightarrow-\infty .
$$

ДоКАЗАТЕЛЬСТво. Для построения $h_{+}(t, \alpha)$ вьполним в $(26)$ последовательно замены

$h=e_{+}(\alpha) y_{1}+e_{-}(\alpha) y_{2}, \quad y_{1}, y_{2} \in \mathbb{R}, \quad y_{1}=\left(1+v_{1}\right) \exp \left(\lambda_{+}(\alpha) t\right), \quad y_{2}=v_{2} \exp \left(\lambda_{+}(\alpha) t\right)$.

В результате получаем систему

$$
\begin{aligned}
& \dot{v}_{1}=b_{11}(t, \alpha)\left(1+v_{1}\right)+b_{12}(t, \alpha) v_{2}, \\
& \dot{v}_{2}=b_{21}(t, \alpha)\left(1+v_{1}\right)+\left(b_{22}(t, \alpha)-\lambda_{-}(\alpha)-\lambda_{+}(\alpha)\right) v_{2},
\end{aligned}
$$

для коэффициентов $b_{i j}(t, \alpha), i, j=1,2$, которой в силу (21) имеем

$$
b_{i j}=O\left(\exp \left( \pm \lambda_{ \pm} t\right)\right), \quad t \rightarrow \mp \infty .
$$

Задача же о нахождении решений этой системы, стремящихся к 0 при $t \rightarrow-\infty$, эквивалентна разрешимости системы

$$
\begin{aligned}
& v_{1}=\int_{-\infty}^{t}\left(b_{11}(s, \alpha)+b_{12}(s, \alpha) v_{2}(s)\right) \exp \left(\int_{s}^{t} b_{11}(\tau, \alpha) d \tau\right) d s \\
& v_{2}=\int_{-\infty}^{t}\left(1+v_{1}(s)\right) b_{21}(s, \alpha) \exp \left(\int_{s}^{t}\left(b_{22}(\tau, \alpha)-\lambda_{-}(\alpha)-\lambda_{+}(\alpha)\right) d \tau\right) d s .
\end{aligned}
$$

Рассмотрим оператор П, порожденньй правьми частями уравнений $(30),(31)$, в пространстве $V\left(t_{0}\right)$ непрерывных и ограниченных при $-\infty<t \leqslant-t_{0}, t_{0}>0$, вектор-функций $v(t)=\operatorname{colon}\left(v_{1}, v_{2}\right)$ с нормой

$$
\|v\|=\sup _{-\infty<t \leqslant-t_{0}}\left(\left|v_{1}(t)\right|+\left|v_{2}(t)\right|\right) .
$$

Учитьвая в (30), (31) асимптотические равенства (29), убеждаемся, что для любых двух функций $v(t), w(t) \in V\left(t_{0}\right)$

$$
\|\Pi v-\Pi w\| \leqslant M \exp \left(-\lambda_{+} t_{0}\right)\|v-w\| .
$$

Поэтому при достаточно большом $t_{0}$ к оператору П применим в пространстве $V\left(t_{0}\right)$ принцип сжимающих отображений, а его единственная неподвижная точка $v(t, \alpha)$ удовлетворяет равенству (также вытекающему из (29))

$$
v(t, \alpha)=O\left(\exp \left(\lambda_{+} t\right)\right), \quad t \rightarrow-\infty .
$$

Аналитичность $v(t, \alpha)$ непосредственно следует из аналитичности по $\alpha$ правых частей уравнений $(30),(31)$.

Существование $h_{-}(t, \alpha)$ устанавливается аналогично. Отметим лишь, что в отличие от $h_{+}(t, \alpha)$ это решение определяется неоднозначно, так как наряду с $h_{-}(t, \alpha)$ условиям леммы будет удовлетворять, например, и функция $h_{-}+h_{+}$. Лемма 2 доказана.

Перед формулировкой следующего утверждения рассмотрим функцию

$$
c(\alpha)=1+\int_{-\infty}^{\infty}\left(b_{11}(s, \alpha)+b_{12}(s, \alpha) v_{2}(s, \alpha)\right) \exp \left(\int_{s}^{\infty} b_{11}(\tau, \alpha) d \tau\right) d s .
$$

Здесь $v_{2}(t, \alpha)$ - компонента решения системы $(30),(31)$, которая, как будет показано ниже, при $t \rightarrow+\infty$ стремится к 0 с экспоненциальной скоростью. 
Лемма 3. При $t \rightarrow+\infty$ справедливы равенства

$$
h_{+}\left(c(\alpha) e_{+}(\alpha)+o(1)\right) \exp \left(\lambda_{+}(\alpha) t\right), \quad h_{-}=O\left(\exp \left(\lambda_{+}(\alpha) t\right)\right) .
$$

ДокАЗАтЕльство. Учитьвая в системе (30), (31) асимптотические формулы (29), без труда убеждаемся, что для компонент $v_{1}(t, \alpha), v_{2}(t, \alpha)$ ее решения справедливы оценки

$$
\begin{gathered}
\max _{-\infty<\tau \leqslant t}\left|v_{1}(\tau, \alpha)\right| \leqslant M_{1} \max _{-\infty<\tau \leqslant t}\left|v_{2}(\tau, \alpha)\right|+M_{2}, \\
\left|v_{2}(t, \alpha)\right| \leqslant M_{3}\left(1+\max _{-\infty<\tau \leqslant t}\left|v_{1}(\tau, \alpha)\right|\right) \omega(t, \alpha),
\end{gathered}
$$

где

$$
\omega(t, \alpha)=\int_{-\infty}^{t}\left|b_{21}(s, \alpha)\right| \exp \left(-\left(\lambda_{-}(\alpha)+\lambda_{+}(\alpha)\right)(t-s)\right) d s,
$$

а постоянные $M_{j}, j=1,2,3$, не зависят от $t \in \mathbb{R}$. Далее, снова привлекая равенства (29), заключаем, что при $t \rightarrow+\infty$

$$
\omega(t, \alpha)= \begin{cases}O(\exp (-r(\alpha) t)), & \lambda_{-}(\alpha)+\lambda_{+}(\alpha) \neq \lambda_{-} \\ O\left(t \exp \left(-\lambda_{-} t\right)\right), & \lambda_{-}(\alpha)+\lambda_{+}(\alpha)=\lambda_{-}\end{cases}
$$

где $r(\alpha)=\min \left\{\lambda_{-}, \lambda_{-}(\alpha)+\lambda_{+}(\alpha)\right\}$. И наконец, объединяя (34)-(36), приходим к вьводу, что при $t \rightarrow+\infty$ функция $v_{2}(t, \alpha)$ стремится к 0 с той же скоростью, что и $\omega(t, \alpha)$.

Установленные свойства $v_{2}(t, \alpha)$ позволяют перейти в $(30)$ к пределу при $t \rightarrow+\infty$ и убедиться в справедливости равенства

$$
\lim v_{1}(t, \alpha)=c(\alpha)-1
$$

эквивалентного первому равенству (33). Второе равенство (33) очевидно, так как норма любого решения системы (26) не может при $t \rightarrow+\infty$ расти быстрее, чем $\exp \left(\lambda_{+}(\alpha) t\right)$. Лемма 3 доказана.

\section{Лемма 4. Справедливы предельные равенства}

$$
\lim _{\alpha \rightarrow+\infty} c(\alpha)=\exp \left(\int_{-\infty}^{\infty} a_{11}(t) d t\right), \quad \lim _{\alpha \rightarrow-\infty} c(\alpha)=\exp \left(\int_{-\infty}^{\infty} a_{22}(t) d t\right)
$$

где $a_{11}(t), a_{22}(t)$ - диагональные әлементы матрицы $A(t)-A_{0}$.

ДокАЗАТЕЛьство. Из структуры матрицы $A_{0}+B(\alpha)$ вытекает, что при $\alpha \rightarrow+\infty$, во-первых, $\lambda_{-}(\alpha), \lambda_{+}(\alpha) \sim \alpha$; во-вторых, ее собственные векторы $e_{+}(\alpha), e_{-}(\alpha)$ стремятся соответственно к $\sigma_{1} e_{1}, \sigma_{2} e_{2}$, где

$$
e_{1}=\operatorname{colon}(1,0), \quad e_{2}=\operatorname{colon}(0,1), \quad \sigma_{j}= \pm 1, \quad j=1,2 .
$$

Отсюда, в свою очередь, следует, что коэффициенты $b_{i j}(t, \alpha)$ системы $(30),(31)$ равномерно по $t \in \mathbb{R}$ стремятся к коэффициентам $a_{i j}(t)$ матрицы $A(t)-A_{0}$, взятьм со знаком "+" или "-"; в частности,

$$
\lim _{\alpha \rightarrow+\infty} b_{11}(t, \alpha)=a_{11}(t)
$$


Из перечисленных вьше фактов следует, что постоянные $M_{j}, j=1,2,3$, в $(34)$ можно выбрать не зависящими от $\alpha$. Отсюда и из неравенства

$$
\max _{t \in \mathbb{R}} \omega(t, \alpha) \leqslant \frac{M}{\alpha}
$$

справедливого при всех достаточно больших $\alpha$ и вытекающего из явного вида (35) для $\omega(t, \alpha)$, заключаем, что

$$
\lim _{\alpha \rightarrow+\infty} \max _{t \in \mathbb{R}}\left|v_{2}(t, \alpha)\right|=0 .
$$

И наконец, используя (38), (39) в формуле (32), убеждаемся в справедливости первого из равенств (37). Второе же из них устанавливается аналогично. Лемма 4 доказана.

ТЕорема 2. Предположим, что при некотором натуральном $k_{0}$ матрица (18) имеет собственное число с положительной действительной частью $и$

$$
c\left(\alpha_{0}\right) \neq 0
$$

где $\alpha_{0}=k_{0}^{2}\left(d_{2}-d_{1}\right) / 2$. Тогда однородный иикл (3) краевой задачи (1) әкспоненииально неустойчив.

ДоКАЗАТЕЛЬСТВо. Обоснование теоремы сводится, очевидно, к анализу расположения мультипликаторов системы

$$
\dot{h}=\left(A(t, \mu)+B\left(\alpha_{0}\right)\right) h,
$$

получающейся из (19) при $k=k_{0}$ после замены

$$
h \exp \left(\frac{k_{0}^{2}\left(d_{1}+d_{2}\right) t}{2}\right) \rightarrow h .
$$

Отметим, что так как след каждой из матриц (18) отрицателен, то при условиях теоремы матрица $A_{0}-k^{2} D$ имеет вещественные собственные числа $\lambda_{1},-\lambda_{2}, 0<\lambda_{1}<\lambda_{2}$. Поэтому вещественными будут и собственные значения матрицы

$$
A_{0}+B\left(\alpha_{0}\right)=A_{0}-k_{0}^{2} D+\frac{k_{0}^{2}\left(d_{1}+d_{2}\right)}{2} I
$$

а значит, $\alpha_{0} \in \Lambda$.

Как и при доказательстве теоремы 1 , изучим сначала систему (41) при $\mu=0$. С этой целью рассмотрим ее матрицу Коши $U(t, s)$ и положим $V(\mu)=U\left(\tau_{2}(\mu),-\tau_{1}(\mu)\right)$. Так как $\alpha_{0} \in \Lambda$, привлекая введенные выше специальные решения $h_{ \pm}(t, \alpha)$, матрицу $V(\mu)$ можно представить в виде

$$
V(\mu)=W\left(\tau_{2}(\mu), \mu\right) W^{-1}\left(-\tau_{1}(\mu), \mu\right)
$$

где

$$
W(t, \mu)=\left[h_{+}\left(t, \alpha_{0}\right) \exp \left(\lambda_{+}\left(\alpha_{0}\right) \tau_{1}(\mu)\right), h_{-}\left(t, \alpha_{0}\right) \exp \left(-\lambda_{-}\left(\alpha_{0}\right) \tau_{1}(\mu)\right)\right]
$$


Несложные вычисления с использованием асимптотических формул (4), (28), (33) и представления (44) показьвают, что

$$
V(\mu)=\left(\left(\begin{array}{cc}
c\left(\alpha_{0}\right) & 0 \\
0 & 0
\end{array}\right)+o(1)\right) \exp \left(\lambda_{+}\left(\alpha_{0}\right) T(\mu)\right),
$$

где, напомним, $T(\mu)$ - период цикла (3). Отсюда и из неравенства (40) следует, что для ведущего (наибольшего по модулю) собственного числа $\lambda_{\max }(\mu)$ матрицы $V(\mu)$ имеет место асимптотическое равенство

$$
\lambda_{\max }(\mu)=\left(c\left(\alpha_{0}\right)+o(1)\right) \exp \left(\lambda_{+}\left(\alpha_{0}\right) T(\mu)\right)=O\left(\mu^{-\lambda_{+}\left(\alpha_{0}\right) / \lambda_{+}}\right) .
$$

При $\mu>0$ вместо (41) рассмотрим вспомогательную систему

$$
\dot{h}=\left(A_{b c}(t, \mu)+B\left(\alpha_{0}\right)\right) h,
$$

где

$$
A_{b c}(t, \mu)= \begin{cases}A(t, \mu), & t \in\left[-\tau_{1}(\mu), \tau_{2}(\mu)\right], \\ A(t), & t \in \mathbb{R} \backslash\left[-\tau_{1}(\mu), \tau_{2}(\mu)\right],\end{cases}
$$

и остановимся на некоторых свойствах ее коэффициентов. Отметим, во-первых, что для $A_{b c}(t, \mu)-A_{0}$ равномерно по $\mu$ сохраняются асимптотические равенства $(21)$. Действительно, при всех $t \geqslant 0$, например, используя асимптотические представления (4), (21), (23), приходим к цепочке неравенств

$$
\begin{aligned}
\left\|A_{b c}(t, \mu)-A_{0}\right\| \exp \left(\lambda_{-} t\right) \leqslant & \left\|A(t)-A_{0}\right\| \exp \left(\lambda_{-} t\right) \\
& +\mu^{\gamma} \max _{0 \leqslant t \leqslant \tau_{2}(\mu)}\|\Delta(t, \mu)\| \exp \left(\lambda_{-} \tau_{2}(\mu)\right) \leqslant M
\end{aligned}
$$

(при $t \leqslant 0$ ситуация аналогичная). Во-вторых, из (4), (23) следует, что

$$
\int_{-\infty}^{\infty}\left\|A_{b c}(t, \mu)-A(t)\right\| \leqslant M \mu^{\gamma} \ln \frac{1}{\mu} .
$$

Из перечисленных свойств матрищы (49) вытекает, что для системы (48) сохраняют силу леммы $2,3:$ у нее существуют решения $h_{ \pm}\left(t, \alpha_{0}, \mu\right)$ с равномерной по $\mu$ асимптотикой (28) при $t \rightarrow-\infty$ и (33) при $t \rightarrow+\infty$, причем в последнем случае $c(\alpha)$ заменяется на

$$
c\left(\alpha_{0}, \mu\right)=c\left(\alpha_{0}\right)+O\left(\mu^{\gamma} \ln \frac{1}{\mu}\right) .
$$

Отсюда следует, что для этой системы сохраняются и формулы (44)-(47) (с заменой $h_{ \pm}\left(t, \alpha_{0}\right)$ на $\left.h_{ \pm}\left(t, \alpha_{0}, \mu\right)\right)$.

Итак, для ведущего мультипликатора системы (41) справедлива асимптотическая формула (47). Обрашая теперь замену (42) и учитьвая, что (см. (43))

$$
\lambda_{+}\left(\alpha_{0}\right)=\lambda_{1}+\frac{k_{0}^{2}\left(d_{1}+d_{2}\right)}{2}
$$

для ведущего мультипликатора исходной системы (19) (при $k=k_{0}$ ) из (47) вьводим формулу

$$
\lambda_{\max }(\mu)=\left(c\left(\alpha_{0}\right)+o(1)\right) \exp \left(\lambda_{1} T(\mu)\right)=O\left(\mu^{-\lambda_{1} / \lambda_{+}}\right) .
$$

Модуль другого ее мультипликатора в силу теоремы Лиувилля имеет порядок $\mu^{\lambda_{2}} / \lambda_{+}$. Теорема 2 доказана.

Будем говорить, что однородный цикл (3) неустойчив на моде $\cos k x$ с номером $k$, если соответствующая система (19) имеет мультипликатор, модуль которого больше 1. 
ТЕОРема 3. Пусть однородный иикл (3) неустойчив на моде с номером $k_{0}$, $k_{0} \geqslant 2, u$

$$
c\left(\alpha_{k}\right) \neq 0, \quad k=1, \ldots, k_{0}-1,
$$

где $\alpha_{k}=k^{2}\left(d_{2}-d_{1}\right) / 2$. Тогда он будет неустойчивым и на всех модах с номерамu $k \leqslant k_{0}$.

ДокАЗАТЕЛЬСТвО. В силу теоремы 2 проблема сводится к доказательству неравенств

$$
\operatorname{det}\left(A_{0}-k^{2} D\right)<0, \quad k<k_{0} .
$$

Для их обоснования отметим сначала неравенство

$$
\operatorname{det}\left(A_{0}-k_{0}^{2} D\right) \leqslant 0
$$

вытекающее из неустойчивости цикла (3) на моде с номером $k_{0}$ (в противном случае, что ясно из доказательства теоремы 1 , при $k=k_{0}$ была бы устойчивость). Рассмотрим, далее, квадратный трехчлен $P(z)=\operatorname{det}\left(A_{0}-z D\right)$, обладающий свойствами: $P(0)<0$, $P^{\prime \prime}(z)>0$ - и имеющий вследствие этого единственньй положительньй корень $z_{0}$. Так как (см. (53)) $z_{0} \geqslant k_{0}^{2}$, неравенства (52) вытекают автоматически. Теорема 3 доказана.

В дополнение к теоремам 2, 3 остановимся на ряде интересных моментов. Отметим, во-первых, что функция (32) может иметь лишъ изолированные нули, так как она аналитична по $\alpha \in \Lambda$ и не равна 0 тождественно (см. (37)). Поэтому неравенства (40), (51) означают некоторую общность положения, связанную с коэффициентами диффузии.

Во-вторьх, $c(0)=0$. Действительно, при $\alpha=0$ система (26) представляет собой линеаризацию системы $(2)$ при $\mu=0$ на решении $u_{0}(t)$. Поэтому она имеет решение $d u_{0} / d t$, причем (см. условие 1$)$

$$
\frac{d u_{0}}{d t}=O\left(\exp \left(\lambda_{+} t\right)\right), \quad t \rightarrow-\infty, \quad \frac{d u_{0}}{d t}=O\left(\exp \left(-\lambda_{-} t\right)\right), \quad t \rightarrow+\infty .
$$

Так как, очевидно, $h_{+}(t, 0)$ совпадает с $d u_{0} / d t$ с точностью до сдвига по времени, отсюда и следует требуемый факт. Заметим также, что у функции (32) могут быть и другие нули. Например, если предположить, что $c^{\prime}(0) \neq 0, \Lambda=\mathbb{R}$, то в силу (37) у уравнения $c(\alpha)=0$ будет хотя бы один корень $\alpha_{*} \neq 0$.

В-третьих, теоремы 2, 3 также допускают наглядную геометрическую интерпретацию, подобную той, что изложена в п. 2 .

И наконец, в-четвертых, область неустойчивости цикла (3) в плоскости параметров $\left(d_{1}, d_{2}\right)$ всегда не пуста: неустойчивость имеет место, например, при достаточно малых $d_{1}, d_{2}$ таких, что $\left(d_{1}-d_{2}\right) / 2$ не является нулем функции $(32)$. Последнее обстоятельство характерно именно для однородного цикла, бифурцирующего из петли сепаратрисы, так как в рассмотренных в [4]-[6] случаях область может быть и пустой.

4. О синхронизмах. Будем говорить о синхронизме, если однородньй цикл (3) устойчив и в то же время

$$
\operatorname{det}\left(A_{0}-k^{2} D\right)<0, \quad k=1, \ldots, k_{0},
$$

при некотором натуральном $k_{0}$. Из теорем 2,3 следует, что в этом случае величины $c\left(\alpha_{k}\right), k=1, \ldots, k_{0}$, асимптотически малы. Тем самьм, синхронизмы возможны лишш 
при значениях $d_{1}, d_{2}$, лежащих на плоскости $\left(d_{1}, d_{2}\right)$ в асимптотически малых окрестностях прямых $d_{2}-d_{1}=2 \alpha_{*} / k^{2}$, где $k \geqslant 1, \alpha_{*}$ - произвольньй нуль функции (32). В частности, всегда имеется тривиальньй синхронизм $d_{1}=d_{2}$, так как в этом случае однородный цикл (3), очевидно, будет устойчивьг. Возможность же реализации нетривиального синхронизма в простейшей ситуации иллюстрирует следующее утверждение.

ТЕОрема 4. Пусть при некоторьх $d_{1}^{0}, d_{2}^{0}>0, d_{1}^{0} \neq d_{2}^{0}$,

$$
\begin{gathered}
\operatorname{det}\left(A_{0}-D_{0}\right)<0, \quad \operatorname{det}\left(A_{0}-k^{2} D_{0}\right)>0, \quad k \geqslant 2, \\
c\left(\alpha_{0}\right)=0, \quad c^{\prime}\left(\alpha_{0}\right) \neq 0,
\end{gathered}
$$

где $D_{0}=\operatorname{diag}\left\{d_{1}^{0}, d_{2}^{0}\right\}, \alpha_{0}=\left(d_{2}^{0}-d_{1}^{0}\right) / 2$. Тогда найдутся непрерывные функиии $d_{j}(\mu), d_{j}(0)=d_{j}^{0}, j=1,2$, такие, что при $d_{j}=d_{j}(\mu), j=1,2$, однородныи иикл $(3)$ краевой задачи (1) әкспоненииально орбитально устойчив.

ДоКАЗАТЕЛЬСТво. Положим в $(1) d_{1}=d_{1}^{0}, d_{2}=d_{2}^{0}+s$, где $|s| \ll 1$, и будем менять параметр $s$ так, чтобы знаки определителей (54) сохранялись, а величина $c\left(\alpha_{0}\right)$ меняла знак. Тогда из формулы (50) следует, что ведущий мультипликатор системы (19) при $k=1$ также меняет знак, а значит, существует такое значение $s=s_{0}(\mu), s_{0}(0)=0$, параметра $s$, при котором мультипликаторы этой системы становятся комплексньми. Остается отметить, что в силу теоремы Лиувилля их модули при $s=s_{0}(\mu)$ приобретают порядок $\mu^{\left(\lambda_{2}-\lambda_{1}\right) /\left(2 \lambda_{+}\right)}$, где $\lambda_{1},-\lambda_{2}, 0<\lambda_{1}<\lambda_{2},-$ собственные числа матрицы $A_{0}-D_{0}$. Теорема 4 доказана.

В заключение остановимся на геометрическом смысле описанного синхронизма. Можно показать, что при условиях $(54),(55)$ и при $\mu=0$ в достаточно малой окрестности петли $\Gamma_{0}$ у системы (1) существует двумерное многообразие решений, стремящихся $\mathrm{K}$ нулевому состоянию равновесия при $t \rightarrow \pm \infty$. Таким образом, в этом случае наблюдается определенная "возвращаемость" траекторий к седлу, которая несмотря на наличие у последнего пространственно неоднородного неустойчивого направления гарантирует устойчивость цикла (3). Если же $c\left(\alpha_{0}\right) \neq 0$, то подобной возвращаемости нет и цикл $(3)$ оказьвается неустойчивьп.

\section{СПИСОК ЦИТИРОВАННОЙ ЛИТЕРАТУРЫ}

[1] Иваницкий Г. Р., Кринский В. И., Сельков Е. Е. Математическая биофизика клетки. М.: Наука, 1978.

[2] Колесов Ю.С.Проблема адекватности экологических уравнений // Деп. ВИНИТИ №1901-85. М.: ВИНИТИ 1985.

[3] Гленсдорф П., Пригожин И. Термодинамическая теория структуры, устойчивости и флюктуаций. М.: Мир, 1973.

[4] Колесов А. Ю. Устойчивость однородного цикла в системах с диффузией. Дисс. ... к. ф.-м. н. М.: МИАН, 1987.

[5] Мищенко Е.Ф., Колесов Ю. С., Колесов А. Ю., Розов Н. Х. Периодические движения и бифуркационные процессы в сингулярно возмущенных системах. М.: Физматлит, 1995.

[6] Mishchenko E.F., Kolesov Yu.S., Kolesov A. Yu., Rozov N. Kh. Asymptotic Methods in Singularly Perturbed Systems. New York-London: Plenum, 1994.

[7] Андронов А. А., Леонтович Е. А., Гордон И. И., Майер А. Г. Теория бифуркаций динамических систем на плоскости. М.: Наука, 1967.

[8] Хартман Ф. Обыкновенные дифференциальные уравнения. М.: Мир, 1970. 